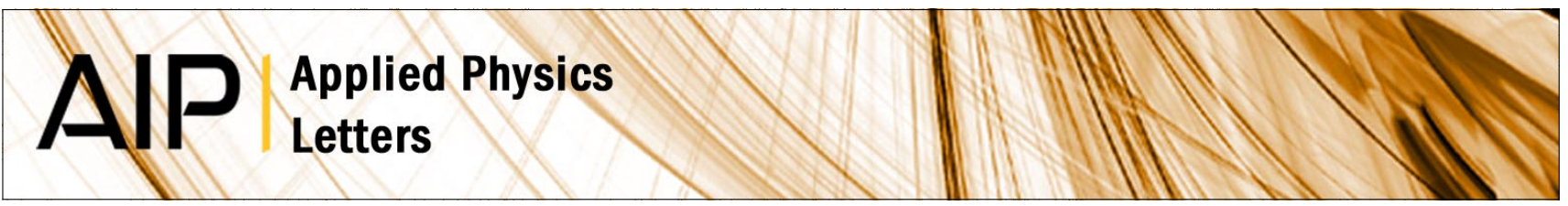

\title{
Electrical detection of single-base DNA mutation using functionalized nanoparticles
}

Mohammud R. Noor, Swati Goyal, Shawn M. Christensen, and Samir M. Iqbal

Citation: Appl. Phys. Lett. 95, 073703 (2009); doi: 10.1063/1.3152768

View online: http://dx.doi.org/10.1063/1.3152768

View Table of Contents: http://apl.aip.org/resource/1/APPLAB/v95/i7

Published by the American Institute of Physics.

\section{Related Articles}

Nanoscale structure of protamine/DNA complexes for gene delivery

Appl. Phys. Lett. 102, 053703 (2013)

Perspective: Nanomotors without moving parts that propel themselves in solution

J. Chem. Phys. 138, 020901 (2013)

Micro-/nanofluidics based cell electroporation

Biomicrofluidics 7, 011301 (2013)

Amplified spontaneous emission of Rhodamine $6 \mathrm{G}$ embedded in pure deoxyribonucleic acid

Appl. Phys. Lett. 101, 171113 (2012)

Single particle demultiplexer based on domain wall conduits

Appl. Phys. Lett. 101, 142405 (2012)

\section{Additional information on Appl. Phys. Lett.}

Journal Homepage: http://apl.aip.org/

Journal Information: http://apl.aip.org/about/about_the_journal

Top downloads: http://apl.aip.org/features/most_downloaded

Information for Authors: http://apl.aip.org/authors

\section{ADVERTISEMENT}

\section{AIP Applied Physics Letters}

\section{EXPLORE WHAT'S NEW IN APL}

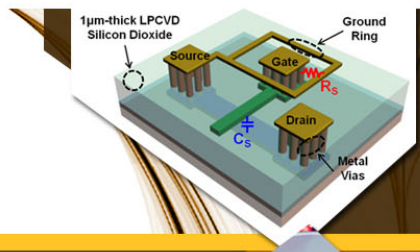

SURFACES AND INTERFACES

Focusing on physical, chemical, biological, structural, optical, magnetic and electrical properties of surfaces and interfaces, and more.. 


\title{
Electrical detection of single-base DNA mutation using functionalized nanoparticles
}

\author{
Mohammud R. Noor, ${ }^{1,2}$ Swati Goyal, ${ }^{2,3}$ Shawn M. Christensen, ${ }^{4}$ and Samir M. Iqbal ${ }^{1,2,5, a)}$ \\ ${ }^{1}$ Department of Electrical Engineering, The University of Texas at Arlington, Arlington, \\ Texas 76019, USA \\ ${ }^{2}$ Nanotechnology Research and Teaching Facility, The University of Texas at Arlington, Arlington, \\ Texas 76019, USA \\ ${ }^{3}$ Department of Bioengineering, The University of Texas at Arlington, Arlington, Texas 76019, USA \\ ${ }^{4}$ Department of Biology, The University of Texas at Arlington, Arlington, Texas 76019, USA \\ ${ }^{5}$ Joint Graduate Committee of Biomedical Engineering Program, University of Texas at Arlington and \\ University of Texas Southwestern Medical Center at Dallas, Arlington, Texas 76019 USA
}

(Received 25 March 2009; accepted 18 May 2009; published online 17 August 2009)

\begin{abstract}
We report an electrical scheme to detect specific DNA. Engineered hairpin probe DNA are immobilized on a silicon chip between gold nanoelectrodes. Hybridization of target DNA to the hairpin melts the stem nucleotides. Gold nanoparticle-conjugated universal reporter sequence detects the open hairpins by annealing to the exposed stem nucleotides. The gold nanoparticles increase charge conduction between the electrodes. Specifically, we report on a hairpin probe designed to detect a medically relevant mutant form of the $K$-ras oncogene. Direct current measurements show three orders of magnitude increase in conductivity for as low as 2 fmol of target molecules. (C) 2009 American Institute of Physics. [DOI: 10.1063/1.3152768]
\end{abstract}

Individual genetic mutations can predispose a cell or tissue toward cancer. High throughput screening has identified altered genes in many spontaneous as well as hereditary cancers. ${ }^{1,2}$ For example, mutations in base 12 of the $K$-ras gene are implicated in over $90 \%$ of pancreatic carcinomas, and it is also considered a prognostic indicator for lung cancer patients. ${ }^{3}$ Specific mutations in such genes can thus be used as diagnostic indicators for the susceptibility of disease, aiding in early detection and treatment. ${ }^{4}$

Microarrays are a common method to screen for the presence or absence of particular genomic sequences. Microarrays use single stranded DNA probes attached to chips to capture fluorescently tagged genomic DNA. The fluorescent markers are excited for detection. Fluorescent tags, however, can affect the stability and fidelity of the probetarget interactions, thereby reducing reliability. ${ }^{5}$ To overcome this (and other) inherent limitation(s), we report an alternative probe and detection method-hairpin DNA probe instead of single stranded linear DNA, and electrical detection instead of fluorescent detection, with single-base mismatch sensitivity down to $2 \mathrm{fmol}$ of target DNA. Previous experiments along these lines have demonstrated the potential of hairpin probes to improve reliability and fidelity of microarrays. In addition, a number of alternative detection mechanisms have been reported with the detection limits between a few nmol and $1.8 \mathrm{fmol}^{6-8}$ impedance measurements, ${ }^{9}$ capacitance detection, ${ }^{10}$ cyclic voltammeter measurements, ${ }^{11}$ and calorimetric measurements.

We attach a hairpin probe to a silicon oxide surface located between two isolated nanoelectrodes (Fig. 1). The loop of the hairpin and one stem forming segment is complementary to the DNA of interest. The other side of the hairpin stem is complementary to an engineered reporter oligonucleotide (called GNP-reporter). The GNP-reporter is conjugated

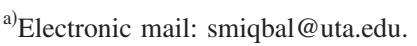

to a gold-nanoparticle (GNP). When the DNA of interest hybridizes to the loop, the stem of the hairpin probe destabilizes and melts. Electrical detection of the hairpin opening is conferred upon the system by the ability of the GNP-reporter to conduct electricity between the nanoelectrodes upon annealing to the exposed stem.

The DNA oligonucleotides used in this study (Table I) were purchased from Sigma Aldrich (Saint Louis, MO). The oligonucleotides are given the names "hairpin probe," "PC-target," "MM-target," and "GNP-reporter" based on their roles. Hairpin probe consists of a three nucleotide spacer, a 12 nucleotide loop, and a six base pair (bp) stem. The target DNA, PC-target and MM-target, are identical to

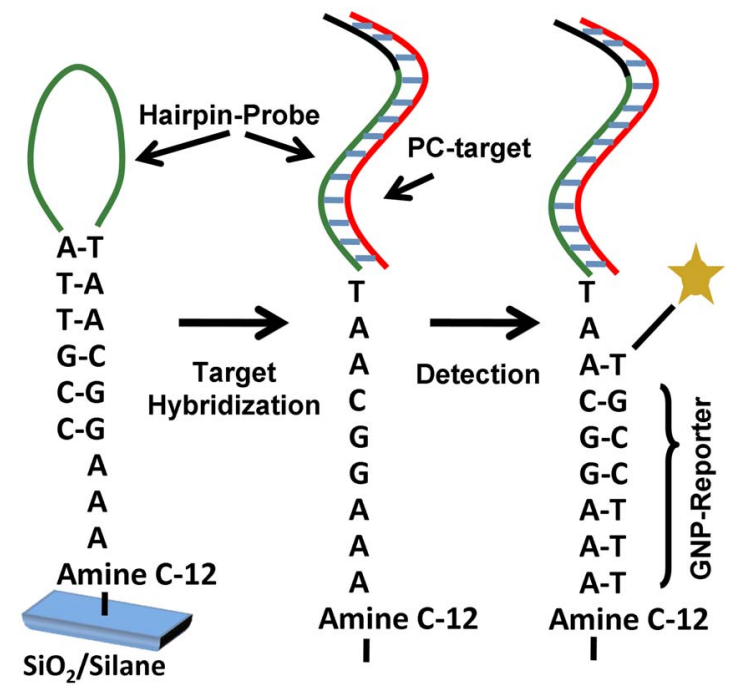

FIG. 1. (Color online) Schematics showing reporter sequence carrying the gold nanoparticle (yellow star) binding to the hairpin probe after complementary DNA (PC-target, red) interacts with the loop (green) of the hairpin. The PC-target starts with GGCAA and covers the whole loop. Black portion depicts the part of hairpin that formed the stem before opening. 
TABLE I. DNA sequences and their modifications.

\begin{tabular}{lll}
\hline \hline Sequence Name & \multicolumn{1}{c}{ Oligonucleotide $\left(5^{\prime}\right.$ to $\left.3^{\prime}\right)$} & \multicolumn{1}{c}{ Modification } \\
\hline Hairpin Probe & AAAGGCAATTTCGCCGCCGCCATTGCC & $5^{\prime}$ C12 Amine \\
PC-target & GGC AAT GGC GGC GGC GAA & None \\
MM-target & GGC AGT GGC GGC GGC GAA & None \\
GNP-reporter & TGCCTTT & $5^{\prime}$ Thiol and GNP \\
\hline
\end{tabular}

each other except at one position (marked in bold in Table I). PC-target, the mutated version of the oncogene, is perfectcomplementary to the hairpin probe loop and one stem forming side. MM-target is the $18 \mathrm{bp}$ wild type DNA taken from $K$-ras oncogene. The MM-target is unable to open and hybridize the hairpin probe while PC-target (the mutant) opens the hairpin structure and forms a duplex (Fig. 1). The seven base GNP-reporter increases the detection limit of the system by increasing electrical conductance across the nanoelectrodes when the hairpin is in the open configuration. Our approach can be used, in principle, to electrically detect any specific oncogene or mutation.

Thermodynamically, the probe DNA forms a strong hairpin loop structure with stem size of six bp having $\Delta G_{\text {probe }}$ of $-4.42 \mathrm{kcal} / \mathrm{mol}$ at $25{ }^{\circ} \mathrm{C} .{ }^{12} \mathrm{PC}$-target binds to the probe molecule with $\Delta G_{\mathrm{PC}}$ of $-24.45 \mathrm{kcal} / \mathrm{mol}$. MM-target has calculated $\Delta G_{\mathrm{MM}}$ of $-21.20 \mathrm{kcal} / \mathrm{mol}$. The reduced $\Delta G$ for MM fails to overcome the hairpin conformation, indicating hairpin structure has a lot more free energy penalty to overcome for a MM than what is calculated by standard formulas. The equilibrium equations associated with $\mathrm{PC}$ and MM interactions with a hairpin probe in closed loop form $(\mathrm{PR})$ can be written as $\mathrm{PC}+\mathrm{PR} \rightarrow \mathrm{PC}_{\text {duplex }}$ and $\mathrm{MM}+\mathrm{PR}$ $\rightarrow \mathrm{MM}_{\text {duplex }}$ (with respective reaction free energies $\Delta G_{\mathrm{PC}}$ and $\left.\Delta G_{\mathrm{MM}}\right)^{10}$

The equilibrium constants for $\mathrm{PC}\left(K_{\mathrm{PC}}\right)$ and $\mathrm{MM}\left(K_{\mathrm{MM}}\right)$ can be calculated from $\ln K_{\mathrm{PC}}=\ln \left(\left[\mathrm{PC}_{\text {duplex }}\right] /[\mathrm{PC}][\mathrm{PR}]\right)$ $=-\left(\Delta G_{\mathrm{PC}} / R T\right)$ and $\ln K_{\mathrm{MM}}=\ln \left(\left[\mathrm{MM}_{\text {duplex }}\right] /[\mathrm{MM}][\mathrm{PR}]\right)$ $=-\left(\Delta G_{\mathrm{MM}} / R T\right)$. Setting $[\mathrm{MM}]=[\mathrm{PC}]$ yields the energy criteria $-\Delta G_{\mathrm{PC}}<0$ and $-\Delta G_{\mathrm{MM}}>0$. This model is directly verified with the introduction of GNP-reporter as a way to measure inability of MM to open the hairpin probe.

The chips with nanoelectrodes were fabricated in two steps of lithography. On the first layer $\mathrm{Ti} / \mathrm{Au}$ (thickness $50 / 150 \AA$ ) metal pads $500 \mathrm{~nm}$ apart were made using e-beam lithography and lift-off. In the second step, optical lithography was done to fabricate probing pads to contact the nanoelectrodes. Bare silicon chips were used to test the attachment and detection schemes prior to using nanoelectrode chips.

The chips were cleaned in oxygen plasma at $200 \mathrm{~W}$ in $\mathrm{Ar}+\mathrm{O}^{2}$ and Piranha solution, followed by surface attachment of probe DNA in a nitrogen glovebox with controlled ambi-

TABLE II. Count of average number of GNPs on silicon surfaces.

\begin{tabular}{lcc}
\hline \hline \multicolumn{1}{c}{ DNA Complexes } & $\begin{array}{c}\text { Mean number } \\
\text { of particles } / \mu \mathrm{m}^{2}\end{array}$ & $\begin{array}{c}\text { Standard } \\
\text { deviation }\end{array}$ \\
\hline Hairpin Probe+PC-target+GNP-reporter & 11.62 & 5.60 \\
Hairpin Probe+MM-target+GNP-reporter & 0.86 & 0.76 \\
Hairpin Probe+GNP-reporter (Control) & 0.10 & 0.09 \\
Silane SAM+GNP-reporter (Control) & 0.01 & 0.00 \\
\hline \hline
\end{tabular}

ence and temperature, as previously reported. ${ }^{13}$ The surfaceattached probe molecules were heat cycled in TE buffer three times to ensure all molecules formed hairpin loop structures. The GNP-reporter conjugates were prepared using thiol-gold chemistry. ${ }^{14}$ The functionalized chips were immersed in TE buffer (pH 7.4) containing target DNA, either PC-target or MM-target, at a concentration of $2 \mathrm{fmol} / \mu \mathrm{l}$ for $24 \mathrm{~h}$ at $40{ }^{\circ} \mathrm{C}$, followed by rinse with methanol and de-ionized water. Chips were then incubated in TE solution containing GNP-reporter. The GNP-reporter binds to the stem region of the probe toward the chip surface when it becomes available on binding of PC with probe (and is unavailable otherwise). In addition to the "PC" and "MM" chips, two control chips were used: (1) probe-functionalized chips not exposed to any target and (2) chips lacking probe and exposure to target DNA (i.e., silane SAM surface chemistry only). Control chips were incubated with GNP-reporter sequence as above. Neither control chip showed attachment of the GNP-reporter sequence.

The GNPs on the surface of control, MM, and PC exposed bare silicon chips were visualized by scanning electron microscopy (SEM) and manually counted using ImageJ software. ${ }^{15}$ The GNP count data were normalized as number of GNPs per square micron in Table II. The data clearly shows an order difference in the number of GNPs captured by the probe in case of PC-target than for MM-target, and a negligible number for control chips (Inset to Fig. 2).

Probing of control, MM, and PC nanoelectrode chips with the Agilent $4155 \mathrm{C}$ semiconductor parameter analyzer with a sweep of -1 to $+1 \mathrm{~V}$ showed a significant difference between the PC and MM chips (Fig. 2). The current-voltage $(I-V)$ measurements on MM chips showed a slight increase in conductivity (within the same order) in $\sim 3 \%$ of the nano-

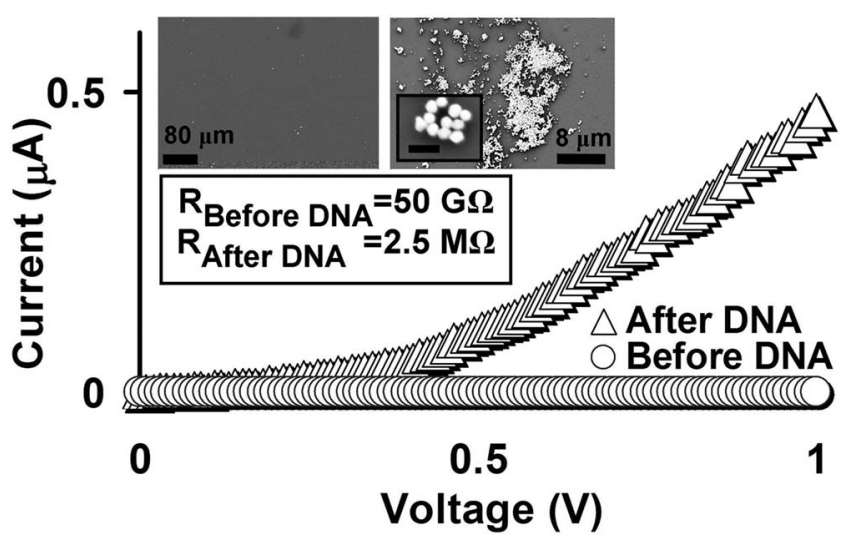

FIG. 2. Comparison of $I-V$ data for a representative nanogap before hybridization of PC-target and probe (circles) and after exposure and hybridization of probe to PC-DNA and GNP-reporter (triangles). Insets: Left image shows chip with probe + MM-target + GNP-reporter. Right image shows chip with probe + PC-target + GNP-reporter. The inset to right micrograph shows the details of GNP-reporter payload on bare silicon chips (scale bar: $400 \mathrm{~nm}$ ). 
electrode pairs. On the other hand, more than $70 \%$ of nanoelectrode pairs showed a robust increase in conductivity on the PC-exposed chips. There were three orders of reduction in the resistance between the nanoelectrodes for PC-DNA (and amplified by GNP-reporter). The presence of GNPs provide charge-hopping sites to carriers, thus acting as a transduction block for the binding of PC with the probe. The tunneling current in a system of the nanogap electrodes with insulator (vacuum) between them can be approximately described by the Simmons formula as

$J=\left(\frac{\alpha}{\delta_{z}^{2}}\right)\left\{\bar{\varphi} \exp \left(-A \delta_{z} \sqrt{\bar{\varphi}}\right)-(\bar{\varphi}+e V) \exp \left[-A \delta_{z} \sqrt{\bar{\varphi}+e V}\right]\right\}$

where $\alpha=e /\left(4 \pi^{2} \beta^{2} \hbar\right), A=2 \beta \sqrt{2 m / \hbar^{2}}, \bar{\varphi}$ is the average barrier height relative to Fermi level of the negative electrode, $\delta_{z}$ is the barrier width, and $e V$ represents the applied voltage across the nanoelectrodes. ${ }^{16} \beta$ is the dimensionless correction factor, $e$ and $m$ are the charge and mass of electron respectively, and $\hbar$ is Dirac's constant. At small voltages when $\bar{\varphi} \gg e V$, the Simmons formula simplifies as $J=\left(\gamma \sqrt{\bar{\varphi} V} / \delta_{z}\right) \exp \left(-A \delta_{z} \sqrt{\bar{\varphi}}\right)$, where $\gamma$ $=(e \sqrt{2 m}) /\left(4 \beta \pi^{2} \hbar^{2}\right)$. In the case of small voltages, the barrier height $\bar{\varphi}$ becomes independent of the applied voltage and the tunneling current becomes linearly dependent only on the applied voltage. ${ }^{17}$ The tunneling current characteristic can thus be modeled as two electrodes with high resistance between them. As the PC-target binds between these electrodes and GNP-reporter brings in GNPs, electrons find a lower barrier and thus start tunneling efficiently. Such framework shows the potential of electrical conductivity in microarrays.

The SEM imaging of the MM and PC nanoelectrode chips also confirmed the validity of the $I-V$ measurements (Fig. 3). The images showed clusters of GNPs on PC-target chips between the nanoelectrodes, which provided the pathways for increased current through otherwise insulating electrodes. SEM micrographs also showed a negligible number of GNPs on the surface of MM-DNA chips. The few GNPs on the MM chips can be attributed to physical adsorption.

In summary, we present that the DNA-of-interest target molecules can be electrically detected using engineered hairpin probes. The detection of hybridization events can be amplified by using a GNP-reporter sequence complementary to stem region of the hairpin probe. The presence of GNP between electrodes enhances the conductivity between otherwise insulated nanoelectrodes. The GNP-reporter can be modeled as a circuit breaker demonstrating electrical quantification of the molecular interactions. The device has the
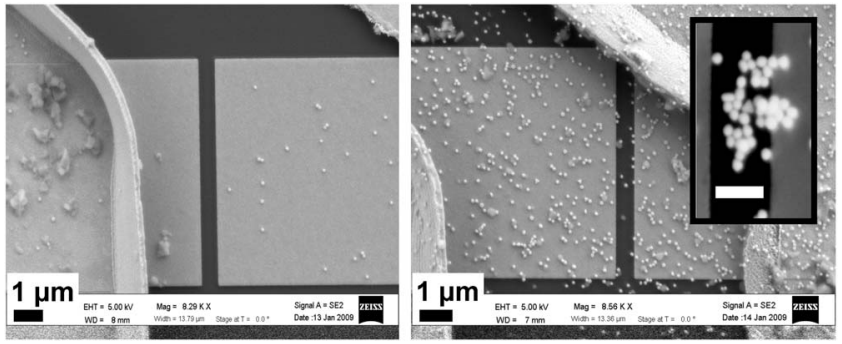

FIG. 3. SEM micrographs of nanoelectrode chips show side by side comparison of the amount of GNP in case of MM-target (left) and PC-target (right). There is a distinct difference in the GNP densities. Inset to right image shows closeup images of GNPs bridging the gap between the nanoelectrodes, causing the increase in conductivity for PC-target (scale bar: $400 \mathrm{~nm}$ )

potential to function as a traditional microarray but without the need to directly tag either the probe or target molecules.

This work was supported by funds from Research Enhancement Program at University of Texas at Arlington and National Science Foundation CAREER grant to S.M.I. (ECCS 0845669). The authors would like to thank Fatima Z. Amir for help with electron microscopy. Mohammud R. Noor and Swati Goyal contributed equally to this work.

${ }^{1}$ K. L. Gorringe, S. Jacobs, E. R. Thompson, A. Sridhar, W. Qiu, D. Y. H. Choong, and I. G. Campbell, Clin. Cancer Res. 13, 4731 (2007).

${ }^{2}$ P. A. Futreal, L. Coin, M. Marshall, T. Down, T. Hubbard, R. Wooster, N. Rahman, and M. R.. Stratton, Nat. Rev. Cancer 4, 177 (2004).

${ }^{3}$ M. Huncharek, J. Muscat, and J.-F. Geschwind, Carcinogenesis 20, 1507 (1999).

${ }^{4}$ R. Etzioni, N. Urban, S. Ramsey, M. McIntosh, S. Schwartz, B. Reid, J. Radich, G. Anderson, and L. Hartwell, Nat. Rev. Cancer 3, 243 (2003).

${ }^{5}$ E. E. Merkina and K. R. Fox, Biophys. J. 89, 365 (2005).

${ }^{6}$ Y. S. Liu, P. P. Banada, S. Bhattacharya, A. K. Bhunia, and R. Bashir, Appl. Phys. Lett. 92, 143902 (2008).

${ }^{7}$ J. Zhang, S. Song, L. Wang, D. Pan, and C. Fan, Nat. Protoc. 2, 2888 (2007).

${ }^{8}$ S. R. Mikkelsen, Electroanalysis 8, 15 (1996).

${ }^{9}$ I. Willner and M. Zayats, Angew. Chem., Int. Ed. 46, 6408 (2007).

${ }^{10}$ F. Wei, B. Sun, W. Liao, J. Ouyang, and X. Sheng Zhao, Biosens. Bioelectron. 18, 1149 (2003).

${ }^{11}$ Y. Jin, X. Yao, Q. Liu, and J. Li, Biosens. Bioelectron. 22, 1126 (2007).

${ }^{12}$ Gene Runner Software, version 3.01, Hastings Software, www.generunner.net.

${ }^{13}$ S. M. Iqbal, D. Akin, and R. Bashir, Nat. Nanotechnol. 2, 243 (2007).

${ }^{14}$ J. J. Storhoff, R. Elghanian, R. C. Mucic, C. A. Mirkin, and R. L. Letsinger, J. Am. Chem. Soc. 120, 1959 (1998).

${ }^{15}$ ImageJ Software, National Institutes of Health, USA, http:// rsb.info.nih.gov/ij/.

${ }^{16}$ W. Wang, T. Lee, and M. A. Reed, Phys. Rev. B 68, 035416 (2003).

${ }^{17}$ John G. Simmons, J. Appl. Phys. 34, 1793 (1963). 